\title{
Multiple Positive Periodic Solutions for a Functional Difference System
}

\author{
Yue-Wen Cheng and Hui-Sheng Ding \\ College of Mathematics and Information Science, Jiangxi Normal University, Nanchang, Jiangxi 330022, China \\ Correspondence should be addressed to Hui-Sheng Ding; dinghs@mail.ustc.edu.cn
}

Received 20 January 2014; Accepted 12 May 2014; Published 22 May 2014

Academic Editor: Samir Saker

Copyright ( 2014 Y.-W. Cheng and H.-S. Ding. This is an open access article distributed under the Creative Commons Attribution License, which permits unrestricted use, distribution, and reproduction in any medium, provided the original work is properly cited.

We obtain two existence results about multiple positive periodic solutions for a class of functional difference system. Two examples are given to illustrate our results.

\section{Introduction and Preliminaries}

Throughout this paper, we denote by $\mathbb{Z}$ the set of all integers, by $\mathbb{R}$ the set of all real numbers, and by $X$ a real Banach space. Moreover, let

$$
\mathbb{R}_{+}^{n}=\left\{\left(x_{1}, \ldots, x_{n}\right): x_{1}, \ldots, x_{n} \geq 0\right\}
$$

and let $l_{T}^{\infty}\left(\mathbb{Z}, \mathbb{R}^{n}\right)\left(l_{T}^{\infty}\left(\mathbb{Z}, \mathbb{R}_{+}^{n}\right)\right)$ be the space of all $T$-periodic functions $f: \mathbb{Z} \rightarrow \mathbb{R}^{n}\left(f: \mathbb{Z} \rightarrow \mathbb{R}_{+}^{n}\right)$, where $T>1$ is fixed positive integer. It is well known that $l_{T}^{\infty}\left(\mathbb{Z}, \mathbb{R}^{n}\right)$ is a Banach space under the norm

$$
\|f\|=\max _{1 \leq k \leq T 1 \leq j \leq n} \max _{j}\left|f_{j}(k)\right|
$$

where $f=\left(f_{1}, f_{2}, \ldots, f_{n}\right)^{T}$.

The aim of this paper is to investigate the existence of multiple positive periodic solutions to the following functional difference system:

$$
x(k+1)-x(k)=A(k) x(k)+f\left(k, x_{k}\right), \quad k \in \mathbb{Z},
$$

where $x: \mathbb{Z} \rightarrow \mathbb{R}^{n}$ is an $n$-dimensional vector function

$$
A(k)=\operatorname{diag}\left[a_{1}(k), a_{2}(k), \ldots, a_{n}(k)\right],
$$

$a_{j}, j=1,2, \ldots, n$, are $T$-periodic functions from $\mathbb{Z}$ to $\mathbb{R}, f$ is a function from $\mathbb{Z} \times l_{T}^{\infty}\left(\mathbb{Z}, \mathbb{R}^{n}\right)$ to $\mathbb{R}^{n}$, and $x_{k}$ is defined by $x_{k}(m)=x(k+m)$ for all $m \in \mathbb{Z}$.
The existence of periodic solutions has been an important topic in the qualitative theory of functional differential equations and functional difference equations. There is a large body of literature on this interesting topic. We refer the reader to [1-17] and references therein for some recent contributions. Especially, the existence of periodic solutions for system (3) and its variants has been of great interest for many authors (see, e.g., $[5,6,8,9,17]$ and references therein).

It is needed to note that Raffoul [8] and Raffoul and Tisdell [9] have made an important contribution to this topic. In fact, Raffoul constructed Green function for system (3) and transformed system (3) into an equivalent system. This enables us to use some suitable fixed point theorems to investigate the existence of periodic solutions for system (3). In addition, we would like to draw the reader's attention to [6], where Dix et al. initiated the study on the multiple periodic solutions for a variant of system (3) in a 1-dimensional case.

Stimulated by $[6,8,9]$, in this paper, we will make further study on this topic for an $n$-dimensional case. Next, we recall two fixed point theorems, which will be used in the proof of our main results. We first recall some definitions and notations.

A closed convex set $K$ in $X$ is called a cone if the following conditions are satisfied:

(i) if $x \in K$, then $\lambda x \in K$ for any $\lambda \geq 0$,

(ii) if $x \in K$ and $-x \in K$, then $x=0$. 
A nonnegative continuous functional $\psi$ is said to be a concave on $K$ if $\psi$ is continuous and

$$
\begin{array}{r}
\psi(\mu x+(1-\mu) y) \geq \mu \psi(x)+(1-\mu) \psi(y), \\
x, y \in K, \quad \mu \in[0,1] .
\end{array}
$$

Letting $c_{1}, c_{2}, c_{3}$ be three positive constants and letting $\phi$ be a nonnegative continuous functional on $K$, we denote

$$
\begin{gathered}
K_{c_{1}}=\left\{y \in K:\|y\|<c_{1}\right\}, \\
K\left(\phi, c_{1}\right):=\left\{x \in K: \phi(x)<c_{1}\right\}, \\
\overline{K\left(\phi, c_{1}\right)}:=\left\{x \in K: \phi(x) \leq c_{1}\right\}, \\
\partial K\left(\phi, c_{1}\right):=\left\{x \in K: \phi(x)=c_{1}\right\}, \\
K\left(\phi, c_{2}, c_{3}\right)=\left\{y \in K: c_{2} \leq \phi(y),\|y\|<c_{3}\right\} .
\end{gathered}
$$

In addition, we call that $\phi$ is increasing on $K$ if $\phi(x) \geq \phi(y)$ for all $x, y \in K$ with $x-y \in K$.

Lemma 1 (see [18]). Let $K$ be a cone in $X$, let $\alpha$ and $\varphi$ be increasing, nonnegative, continuous functionals on $K$, and let $\rho$ be a nonnegative continuous functional on $K$ with $\rho(0)=0$ such that, for some $c>0$ and $M>0$,

$$
\varphi(u) \leq \rho(u) \leq \alpha(u), \quad\|u\| \leq M \varphi(u)
$$

for all $u \in \overline{K(\varphi, c)}$. Suppose that there exists a completely continuous operator $\Phi: \overline{K(\varphi, c)} \rightarrow K$ and $0<a<b<c$ such that

$$
\rho(\lambda u) \leq \lambda \rho(u), \quad \text { for } 0 \leq \lambda \leq 1, u \in \partial K(\rho, b),
$$

and

(i) $\varphi(\Phi u)>c$, for all $u \in \partial K(\varphi, c)$;

(ii) $\rho(\Phi u)<b$, for all $u \in \partial K(\rho, b)$;

(iii) $K(\alpha, a) \neq \emptyset$ and $\alpha(\Phi x)>a$, for all $u \in \partial K(\alpha, a)$.

Then $\Phi$ has at least two fixed points $u_{1}$ and $u_{2}$ belonging to $\overline{K(\varphi, c)}$ such that

$$
\begin{aligned}
& a<\alpha\left(u_{1}\right), \quad \text { with } \rho\left(u_{1}\right)<b, \\
& b<\rho\left(u_{2}\right), \quad \text { with } \varphi\left(u_{2}\right)<c .
\end{aligned}
$$

Lemma 2 (see [19]). Let $K$ be a cone in $X$, let $c_{4}$ be a positive constant, let $\Phi: \bar{K}_{c_{4}} \rightarrow \bar{K}_{c_{4}}$ be a completely continuous mapping, and let $\psi$ be a concave nonnegative continuous functional on $K$ with $\psi(u) \leq\|u\|$ for all $u \in \bar{K}_{c_{4}}$. Suppose that there exist three constants $c_{1}, c_{2}, c_{3}$ with $0<c_{1}<c_{2}<c_{3} \leq c_{4}$ such that

(i) $\left\{u \in K\left(\psi, c_{2}, c_{3}\right): \psi(u)>c_{2}\right\} \neq \emptyset$ and $\psi(\Phi u)>c_{2}$ for all $u \in K\left(\psi, c_{2}, c_{3}\right)$;

(ii) $\|\Phi u\|<c_{1}$ for all $u \in \bar{K}_{c_{1}}$;

(iii) $\psi(\Phi u)>c_{2}$ for all $u \in K\left(\psi, c_{2}, c_{4}\right)$ with $\|\Phi u\|>c_{3}$.

Then $\Phi$ has at least three fixed points $u_{1}, u_{2}, u_{3}$ in $\bar{K}_{c_{4}}$. Furthermore, $\left\|u_{1}\right\| \leq c_{1}<\left\|u_{2}\right\|$ and $\psi\left(u_{2}\right)<c_{2}<\psi\left(u_{3}\right)$.

\section{Main Results}

Throughout the rest of this paper, we assume that the following assumptions for system (3) hold.

(H0) For every $j \in\{1,2, \ldots, n\}, 0<1+a_{j}(k) \leq 1$ for all $k \in \mathbb{Z}$ and

$$
\prod_{k=1}^{T}\left[1+a_{j}(k)\right] \neq 1
$$

(H1) $k \rightarrow f\left(k, x_{k}\right)$ belongs to $l_{T}^{\infty}\left(\mathbb{Z}, \mathbb{R}_{+}^{n}\right)$ whenever $x \in$ $l_{T}^{\infty}\left(\mathbb{Z}, \mathbb{R}_{+}^{n}\right)$.

(H2) For every $L>0$ and $\varepsilon>0$, there exists a $\delta>0$ such that

$$
\left\|f\left(k, \phi_{k}\right)-f\left(k, \psi_{k}\right)\right\|<\varepsilon, \quad k=1,2, \ldots, T,
$$

for all $\phi, \psi \in l_{T}^{\infty}\left(\mathbb{Z}, \mathbb{R}_{+}^{n}\right)$ with $\|\phi\| \leq L,\|\psi\| \leq L$, and $\|\phi-\psi\|<\delta$.

Now, we define

$$
G_{j}(k, s)=\frac{\prod_{m=s-T+1}^{k-1}\left[1+a_{j}(m)\right]}{1-\prod_{m=1}^{T}\left[1+a_{j}(m)\right]}, \quad j=1,2, \ldots, n,
$$

for $(k, s) \in \mathbb{Z} \times \mathbb{Z}$ with $k \leq s \leq k+T-1$.

Then, by a proof similar to [8], we can transform (3) into the following equivalent equation:

$$
x(k)=\sum_{s=k}^{k+T-1} G(k, s) f\left(s, x_{s}\right), \quad k \in \mathbb{Z},
$$

where

$$
G(k, s)=\operatorname{diag}\left[G_{1}(k, s), G_{2}(k, s), \ldots, G_{n}(k, s)\right] .
$$

It is easy to see that

$$
G(k, s)=G(k+T, s+T)
$$

for all $(k, s) \in \mathbb{Z} \times \mathbb{Z}$ with $k \leq s \leq k+T-1$. In addition, it follows from (H0)-(H2) that, for every $j \in$ $\{1,2, \ldots, n\}, G_{j}(\cdot, \cdot)$ has a positive denominator, while the numerator is a positive and increasing function of $s \in[k, k+$ $T-1]$. Thus, for $(k, s) \in \mathbb{Z} \times \mathbb{Z}$ with $k \leq s \leq k+T-1$, we have

$$
\frac{\prod_{m=k-T+1}^{k-1}\left[1+a_{j}(m)\right]}{1-\prod_{m=1}^{T}\left[1+a_{j}(m)\right]}=G_{j}(k, k) \leq G_{j}(k, s),
$$

$G_{j}(k, s) \leq G_{j}(k, k+T-1)=\frac{1}{1-\prod_{m=1}^{T}\left[1+a_{j}(m)\right]}$. 
Letting

$$
\begin{gathered}
p=\min _{1 \leq k \leq T 1 \leq j \leq n} \min _{j}(k, k), \\
q=\max _{1 \leq k \leq T 1 \leq j \leq n} \max _{j}(k, k+T-1),
\end{gathered}
$$

we have

$$
\begin{gathered}
p \leq G_{j}(k, s) \leq q, \quad(k, s) \in \mathbb{Z} \times \mathbb{Z}, k \leq s \leq k+T-1, \\
j=1,2, \ldots, n .
\end{gathered}
$$

Next, we introduce a set

$K=\left\{x \in l_{T}^{\infty}\left(\mathbb{Z}, \mathbb{R}^{n}\right): \min _{1 \leq k \leq T} x_{j}(k) \geq \sigma\left\|x_{j}\right\|, j=1,2, \ldots, n\right\}$,

where $\sigma=p / q$. It is not difficult to verify that $K$ is a cone in $l_{T}^{\infty}\left(\mathbb{Z}, \mathbb{R}^{n}\right)$. Finally, we define an operator $\Phi$ on $K$ by

$$
(\Phi x)(k)=\sum_{s=k}^{k+T-1} G(k, s) f\left(s, x_{s}\right), \quad x \in K, k \in \mathbb{Z} .
$$

Lemma 3. $\Phi$ is an operator from $K$ to $K$.

Proof. Let $x \in K$. By (H1) and $G(k, s)=G(k+T, s+T)$, we get

$$
\begin{aligned}
(\Phi x)(k+T) & =\sum_{s=k+T}^{k+2 T-1} G(k+T, s) f\left(s, x_{s}\right) \\
& =\sum_{s=k}^{k+T-1} G(k+T, s+T) f\left(s+T, x_{s+T}\right) \\
& =\sum_{s=k}^{k+T-1} G(k, s) f\left(s, x_{s}\right)=(\Phi x)(k),
\end{aligned}
$$

for all $k \in \mathbb{Z}$. So $\Phi x \in l_{T}^{\infty}\left(\mathbb{Z}, \mathbb{R}^{n}\right)$.

In addition, for $j=1,2, \ldots, n$, we have

$$
\left\|(\Phi x)_{j}\right\|=\max _{1 \leq k \leq T} \sum_{s=k}^{k+T-1} G_{j}(k, s) f_{j}\left(s, x_{s}\right) \leq q \sum_{s=1}^{T} f_{j}\left(s, x_{s}\right),
$$

where $f_{j}$ is the $j$ th component of $f$. Then, we obtain

$$
\begin{aligned}
(\Phi x)_{j}(k) & =\sum_{s=k}^{k+T-1} G_{j}(k, s) f_{j}\left(s, x_{s}\right) \\
& \geq p \sum_{s=1}^{T} f_{j}\left(s, x_{s}\right) \geq \frac{p}{q}\left\|(\Phi x)_{j}\right\|
\end{aligned}
$$

for all $k \in \mathbb{Z}$. Thus, $\Phi x \in K$. This completes the proof.
2.1. Existence of Two Positive Periodic Solutions of System (3). In this section, we apply Lemma 1 to establish an existence result about two positive periodic solutions of system (3). For convenience, we list some assumptions.

(H3) There exists a constant $c>0$ such that

$$
p \cdot \max _{1 \leq j \leq n} \sum_{s=0}^{T-1} f_{j}\left(s, x_{s}\right)>c \quad \text { for } x \in K \text { with }\|x\|=c .
$$

(H4) There exists a constant $b>0$ such that

$$
q \cdot \max _{1 \leq j \leq n} \sum_{s=0}^{T-1} f_{j}\left(s, x_{s}\right)<b \quad \text { for } x \in K \text { with }\|x\|=b .
$$

(H5) There exists a constant $a>0$ such that

$$
p \cdot \max _{1 \leq j \leq n} \sum_{s=0}^{T-1} f_{j}\left(s, x_{s}\right)>a \quad \text { for } x \in K \text { with }\|x\|=a
$$

Theorem 4. Assume that there exist three constants $a, b, c$ with $0<a<b<c$ such that (HO)-(H5) hold. Then system (3) has at least two positive T-periodic solutions.

Proof. Firstly, by Lemma 3, $\Phi$ is an operator from $K$ to $K$. Secondly, by a proof similar to [9, Lemma 2.5], one can show that $\Phi: K \rightarrow K$ is completely continuous.

Now, we begin to verify that all the assumptions of Lemma 1 hold. Let

$$
\varphi(x)=\rho(x)=\alpha(x)=\|x\|, \quad x \in K
$$

It is clear that $\alpha, \rho$, and $\varphi$ are increasing, nonnegative, continuous functionals on $K$ with $\rho(0)=0$. Moreover, we have

$$
\|x\| \leq \sigma^{-1} \varphi(x), \quad \rho(\lambda x)=\lambda \rho(x),
$$

for all $x \in K$ and $0 \leq \lambda \leq 1$.

Next, we proceed to show that conditions (i)-(iii) of Lemma 1 are also satisfied. For every $x \in \partial K(\varphi, c)$, noting that $\|x\|=\varphi(x)=c$, by $(\mathrm{H} 3)$, we conclude that

$$
\begin{aligned}
\varphi(\Phi x) & =\max _{1 \leq k \leq T 1 \leq j \leq n} \max _{s=k}^{k+T-1} G_{j}(k, s) f_{j}\left(s, x_{s}\right) \\
& \geq p \cdot \max _{1 \leq k \leq T} \max _{1 \leq j \leq n} \sum_{s=k}^{k+T-1} f_{j}\left(s, x_{s}\right) \\
& =p \cdot \max _{1 \leq j \leq n} \sum_{s=0}^{T-1} f_{j}\left(s, x_{s}\right)>c ;
\end{aligned}
$$


that is, condition (i) of Lemma 1 holds. For every $x \in$ $\partial K(\rho, b)$, since $\|x\|=\rho(x)=b$, by $(\mathrm{H} 4)$, we get

$$
\begin{aligned}
\rho(\Phi x) & =\max _{1 \leq k \leq T 1 \leq j \leq n} \max _{s=k}^{k+T-1} G_{j}(k, s) f_{j}\left(s, x_{s}\right) \\
& \leq q \cdot \max _{1 \leq k \leq T} \max _{1 \leq j \leq n} \sum_{s=k}^{k+T-1} f_{j}\left(s, x_{s}\right) \\
& =q \cdot \max _{1 \leq j \leq n} \sum_{s=0}^{T-1} f_{j}\left(s, x_{s}\right)<b ;
\end{aligned}
$$

that is, condition (ii) of Lemma 1 holds. Finally, it is easy to see that

$$
K(\alpha, a)=\{x \in K:\|x\|<a\} \neq \emptyset,
$$

and for every $x \in K(\alpha, a)$, it follows from (H5) that

$$
\alpha(\Phi x) \geq p \cdot \max _{1 \leq j \leq n} \sum_{s=0}^{T-1} f_{j}\left(s, x_{s}\right)>a .
$$

Thus, condition (iii) of Lemma 1 holds.

Now, by applying Lemma 1 , there exist two fixed points $u_{1}, u_{2} \in \overline{K(\varphi, c)}$, which are just two $T$-periodic solutions to system (3). This completes the proof.

Remark 5. In Theorem 4, the two $T$-periodic solutions $u_{1}, u_{2}$ do not equal zero. In fact, according to Lemma 1 , we have

$$
a<\left\|u_{1}\right\|<b<\left\|u_{2}\right\|<c .
$$

Corollary 6. Assume that (HO)-(H2) and (H4) hold. Moreover,

$$
\begin{aligned}
& \lim _{\|x\| \rightarrow+\infty, x \in K^{1 \leq j \leq n}} \max _{s=0}^{T-1} \sum_{j=0} \frac{f_{j}\left(s, x_{s}\right)}{\|x\|}>\frac{1}{p}, \\
& \lim _{\|x\| \rightarrow 0^{+}, x \in K^{1 \leq j \leq n}} \max _{s=0}^{T-1} \frac{f_{j}\left(s, x_{s}\right)}{\|x\|}>\frac{1}{p} .
\end{aligned}
$$

Then system (3) has at least two positive T-periodic solutions.

Proof. By (34), there exists a constant $c>b$ such that (H3) holds. By (35), there exists a constant $a \in(0, b)$ such that (H5) holds. Then, by applying Theorem 4 , we complete the proof.

Next, we present a simple example, which does not aim at generality but illustrates how to use our existence theorem.

Example 7. Consider the following system:

$$
\begin{aligned}
& x_{1}(k+1)-x_{1}(k)=-\frac{1}{2}\left|\sin \frac{\pi k}{2}\right| x_{1}(k)+f_{1}\left(k, x_{k}\right), \\
& x_{2}(k+1)-x_{2}(k)=-\frac{1}{2}\left|\cos \frac{\pi k}{2}\right| x_{2}(k)+f_{2}\left(k, x_{k}\right),
\end{aligned}
$$

where

$$
\begin{aligned}
f_{1} & \left(k, x_{k}\right) \\
& =f_{2}\left(k, x_{k}\right) \\
& =\frac{4\left[x_{1}(k)+x_{2}(k)\right] \exp \left((1 / 384)\left[x_{1}(k)+x_{2}(k)\right]\right)}{1+x_{1}(k)+x_{2}(k)} .
\end{aligned}
$$

We have $n=T=2$,

$$
\begin{gathered}
a_{1}(k)=-\frac{1}{2}\left|\sin \frac{\pi k}{2}\right|, \quad a_{2}(k)=-\frac{1}{2}\left|\cos \frac{\pi k}{2}\right|, \\
G_{1}(k, s)=\frac{\prod_{m=s-1}^{k-1}\left[1+a_{1}(m)\right]}{1-\prod_{m=1}^{2}\left[1+a_{1}(m)\right]} \\
=2 \prod_{m=s-1}^{k-1}\left[1-\frac{1}{2}\left|\sin \frac{\pi m}{2}\right|\right], \\
G_{2}(k, s)=\frac{\prod_{m=s-1}^{k-1}\left[1+a_{2}(m)\right]}{1-\prod_{m=1}^{2}\left[1+a_{2}(m)\right]} \\
=2 \prod_{m=s-1}^{k-1}\left[1-\frac{1}{2}\left|\cos \frac{\pi m}{2}\right|\right], \\
p=\min _{k \in \mathbb{Z}} \min _{1 \leq j \leq 2} \frac{1+a_{j}(k-1)}{1-\prod_{m=1}^{2}\left[1+a_{j}(m)\right]}=1, \\
q=\max _{k \in \mathbb{Z}} \max _{1 \leq j \leq 2} \frac{1}{1-\prod_{m=1}^{2}\left[1+a_{j}(m)\right]}=2, \\
K=\left\{x \in l_{2}^{\infty}\left(\mathbb{Z}, \mathbb{R}_{+}^{2}\right): \min _{k \in \mathbb{Z}} x_{j}(k) \geq \frac{1}{2}\left\|x_{j}\right\|, j=1,2\right\} .
\end{gathered}
$$

It is easy to verify that conditions (H0)-(H2) hold. Since, for $x \in K$,

$$
\begin{aligned}
\max _{1 \leq j \leq 2} \sum_{s=0}^{1} \frac{f_{j}\left(s, x_{s}\right)}{\|x\|} & \\
= & \frac{4\left[x_{1}(0)+x_{2}(0)\right] \exp \left((1 / 384)\left[x_{1}(0)+x_{2}(0)\right]\right)}{\|x\|\left[1+x_{1}(0)+x_{2}(0)\right]} \\
& +\frac{4\left[x_{1}(1)+x_{2}(1)\right] \exp \left((1 / 384)\left[x_{1}(1)+x_{2}(1)\right]\right)}{\|x\|\left[1+x_{1}(1)+x_{2}(1)\right]} \\
\geq & \frac{4 \exp ((1 / 384)\|x\|)}{1+2\|x\|},
\end{aligned}
$$




$$
\begin{aligned}
& \lim _{\|x\| \rightarrow+\infty} \frac{4 \exp ((1 / 384)\|x\|)}{1+2\|x\|}=+\infty, \\
& \lim _{\|x\| \rightarrow 0^{+}} \frac{4 \exp ((1 / 384)\|x\|)}{1+2\|x\|}=4>1,
\end{aligned}
$$

we conclude that (34) and (35) are satisfied. It remains to verify (H4). Letting $b=192$, for all $x \in K$ with $\|x\|=192$, we have

$$
\begin{aligned}
2 \max _{1 \leq j \leq 2} \sum_{s=0}^{1} f_{j}\left(s, x_{s}\right) & \\
= & \frac{8\left[x_{1}(0)+x_{2}(0)\right] \exp \left((1 / 384)\left[x_{1}(0)+x_{2}(0)\right]\right)}{1+x_{1}(0)+x_{2}(0)} \\
& +\frac{8\left[x_{1}(1)+x_{2}(1)\right] \exp \left((1 / 384)\left[x_{1}(1)+x_{2}(1)\right]\right)}{1+x_{1}(1)+x_{2}(1)} \\
\leq & 16 \exp \left(\frac{1}{192}\|x\|\right)=16 e<b,
\end{aligned}
$$

which means that (H4) holds. Therefore, by Corollary 6, we know that system (36) has at least two positive 2-periodic solutions.

Remark 8. In the above example, 0 is obviously a trivial periodic solution for system (36). But by Remark 5, we know that the two positive 2-periodic solutions do not equal zero.

\subsection{Existence of Three Nonnegative Periodic Solutions of} System (3). In [6], Dix et al. investigated the existence of multiple nonnegative periodic solutions for a first order functional difference equation by the Leggett-Williams fixed point theorem. In this section, we will investigate the existence of multiple nonnegative periodic solutions for system (3) by using an idea similar to that of [6]. For convenience, we also list some assumptions.

(H6) There exists a constant $c_{1}>0$ such that

$$
q \cdot \max _{1 \leq j \leq n} \sum_{s=0}^{T-1} f_{j}\left(s, x_{s}\right)<c_{1} \quad \text { for } x \in K \text { with }\|x\| \leq c_{1} .
$$

(H7) There exists a constant $c_{2}>c_{1}>0$ such that

$\frac{p}{n} \cdot \sum_{j=1}^{n} \sum_{s=0}^{T-1} f_{j}\left(s, x_{s}\right)>c_{2} \quad$ for $x \in K$ with $c_{2} \leq\|x\|<\frac{n c_{2}}{\sigma}$.

(H8) There exists a constant $c_{4}>n c_{2} / \sigma:=c_{3}$ such that

$$
q \cdot \max _{1 \leq j \leq n} \sum_{s=0}^{T-1} f_{j}\left(s, x_{s}\right) \leq c_{4} \quad \text { for } x \in K \text { with }\|x\| \leq c_{4} .
$$

Theorem 9. Assume that (HO)-(H2) and (H6)-(H8) hold. Then system (3) has at least three nonnegative $T$-periodic solutions.

Proof. By the proof of Theorem 4, we know that $\Phi$ is an operator from $K$ to $K$ and completely continuous. Let

$$
\psi(x)=\min _{1 \leq k \leq T} \frac{\sum_{j=1}^{n} x_{j}(k)}{n}, \quad x \in K .
$$

It is easy to see that $\psi$ is a concave nonnegative continuous functional on $K$ and $\psi(x) \leq\|x\|$.

Firstly, we show that $\Phi$ maps $\bar{K}_{c_{4}}$ into $\bar{K}_{c_{4}}$. For every $x \in$ $\bar{K}_{c_{4}}$, we have $\|x\| \leq c_{4}$. Combining this with (H8), we get

$$
\begin{aligned}
\|\Phi x\| & =\max _{k \in \mathbb{Z}} \max _{1 \leq j \leq n} \sum_{s=k}^{k+T-1} G_{j}(k, s) f_{j}\left(s, x_{s}\right) \\
& \leq q \cdot \max _{1 \leq j \leq n} \sum_{s=0}^{T-1} f_{j}\left(s, x_{s}\right) \leq c_{4} .
\end{aligned}
$$

Secondly, let us verify condition (i) of Lemma 2. Since $\sigma<$ $1, c_{3}>c_{2}$, then, it is easy to see that the set

$$
\left\{x \in K\left(\psi, c_{2}, c_{3}\right): \psi(x)>c_{2}\right\} \neq \emptyset .
$$

In addition, for every $x \in K\left(\psi, c_{2}, c_{3}\right)$, we have $c_{2} \leq \psi(x) \leq$ $\|x\|<c_{3}=n c_{2} / \sigma$. Then, by (H7), we have

$$
\begin{aligned}
\psi(\Phi x) & =\frac{1}{n} \cdot \min _{1 \leq k \leq T} \sum_{j=1}^{n} \sum_{s=k}^{k+T-1} G_{j}(k, s) f_{j}\left(s, x_{s}\right) \\
& \geq \frac{p}{n} \cdot \sum_{j=1}^{n} \sum_{s=0}^{T-1} f_{j}\left(s, x_{s}\right)>c_{2}
\end{aligned}
$$

which means that condition (i) of Lemma 2 holds.

Thirdly, for every $x \in \bar{K}_{c_{1}}$, since $\|x\| \leq c_{1}$, it follows from (H6) that

$$
\|\Phi x\| \leq q \cdot \max _{1 \leq j \leq n} \sum_{s=0}^{T-1} f_{j}\left(s, x_{s}\right)<c_{1} ;
$$

that is, condition (ii) of Lemma 2 holds.

Finally, for every $x \in K\left(\psi, c_{2}, c_{4}\right)$ with $\|\Phi x\|>c_{3}$, we have $c_{2} \leq\|x\|<c_{4}$ and

$$
q \cdot \max _{1 \leq j \leq n} \sum_{s=0}^{T-1} f_{j}\left(s, x_{s}\right) \geq\|\Phi x\|>c_{3},
$$

which yields that

$$
\sum_{j=1}^{n} \sum_{s=0}^{T-1} f_{j}\left(s, x_{s}\right) \geq \max _{1 \leq j \leq n} \sum_{s=0}^{T-1} f_{j}\left(s, x_{s}\right)>\frac{c_{3}}{q}=\frac{n c_{2}}{p} .
$$

Then, we have

$$
\psi(\Phi x) \geq \frac{p}{n} \cdot \sum_{j=1}^{n} \sum_{s=0}^{T-1} f_{j}\left(s, x_{s}\right)>c_{2} ;
$$

that is, condition (iii) of Lemma 2 holds. 
Now, by Lemma 2, we know that $\Phi$ has at least three fixed points in $\bar{K}_{c_{4}}$, and thus system (3) has at least three nonnegative $T$-periodic solutions.

Corollary 10. Assume that (HO)-(H2) hold and there exists a constant $c_{2}>0$ such that

$$
\frac{p}{n} \cdot \sum_{j=1}^{n} \sum_{s=0}^{T-1} f_{j}\left(s, x_{s}\right)>c_{2} \quad \text { for } \quad c_{2} \leq\|x\|<\frac{n c_{2}}{\sigma} .
$$

Moreover, there hold

$$
\begin{aligned}
& \lim _{\|x\| \rightarrow+\infty, x \in K^{1 \leq j \leq n}} \max _{s=0} \sum^{T-1} \frac{f_{j}\left(s, x_{s}\right)}{\|x\|}<\frac{1}{q}, \\
& \lim _{\|x\| \rightarrow 0^{+}, x \in K^{1 \leq j \leq n}} \max _{s=0}^{T-1} \frac{f_{j}\left(s, x_{s}\right)}{\|x\|}<\frac{1}{q} .
\end{aligned}
$$

Then system (3) has at least three nonnegative T-periodic solutions.

Proof. We only need to verify that (H6) and (H8) hold. Let

$$
\begin{gathered}
\alpha=q \cdot \lim _{\|x\| \rightarrow 0^{+}, x \in K^{1 \leq j \leq n}} \max _{s=0} \sum^{T-1} \frac{f_{j}\left(s, x_{s}\right)}{\|x\|}, \\
\beta=q \cdot \lim _{\|x\| \rightarrow+\infty, x \in K^{1 \leq j \leq n}} \max _{s=0} \sum^{T-1} \frac{f_{j}\left(s, x_{s}\right)}{\|x\|} .
\end{gathered}
$$

Then $\alpha, \beta \in[0,1)$. There exists a constant $\delta \in\left(0, c_{2}\right)$ such that, for all $x \in K$ with $\|x\| \leq \delta$, there holds

$$
q \cdot \max _{1 \leq j \leq n} \sum_{s=0}^{T-1} f_{j}\left(s, x_{s}\right)<\frac{\alpha+1}{2}\|x\| .
$$

Taking $c_{1}=\delta$, (H6) holds. In addition, there exists a constant $M>n c_{2} / \sigma$ such that, for all $x \in K$ with $\|x\| \geq M$, there holds

$$
q \cdot \max _{1 \leq j \leq n} \sum_{s=0}^{T-1} f_{j}\left(s, x_{s}\right)<\frac{\beta+1}{2}\|x\| .
$$

Taking

$$
c_{4}=M+\sup _{x \in K,\|x\| \leq M}\left[q \cdot \max _{1 \leq j \leq n} \sum_{s=0}^{T-1} f_{j}\left(s, x_{s}\right)\right],
$$

(H8) holds.

Next, we also provide a simple example to illustrate our existence theorem.

Example 11. Let $n=T=2, a_{1}, a_{2}$ be the same as in Example 7, and

$$
\begin{aligned}
f_{1}\left(k, x_{k}\right) & =f_{2}\left(k, x_{k}\right) \\
& =\frac{64\left[x_{1}(k)+x_{2}(k)\right]^{2}}{1+\left[x_{1}(k)+x_{2}(k)+x_{1}(k+1)+x_{2}(k+1)\right]^{4}} .
\end{aligned}
$$

By Example 7, we have $p=1, q=2$, and $\sigma=1 / 2$, and (H0)(H2) hold.

By a direct calculation, we get

$$
\begin{aligned}
\max _{1 \leq j \leq 2} & \sum_{s=0}^{1} \frac{f_{j}\left(s, x_{s}\right)}{\|x\|} \\
& =\frac{64\left[x_{1}(0)+x_{2}(0)\right]^{2}+64\left[x_{1}(1)+x_{2}(1)\right]^{2}}{\|x\| \cdot\left[1+\left(x_{1}(0)+x_{2}(0)+x_{1}(1)+x_{2}(1)\right)^{4}\right]} \\
& \leq \frac{512\|x\|}{1+\|x\|^{4}} .
\end{aligned}
$$

Then, it is easy to see that (53) holds.

Let $c_{2}=1 / 16$. Then, for all

$$
\frac{1}{16} \leq\|x\| \leq \frac{1}{4}=\frac{n c_{2}}{\sigma},
$$

we have

$$
\begin{aligned}
\frac{p}{n} \cdot \sum_{j=1}^{2} & \sum_{s=0}^{1} f_{j}\left(s, x_{s}\right) \\
& =\frac{64\left[x_{1}(0)+x_{2}(0)\right]^{2}+64\left[x_{1}(1)+x_{2}(1)\right]^{2}}{1+\left[x_{1}(0)+x_{2}(0)+x_{1}(1)+x_{2}(1)\right]^{4}} \\
& \geq \frac{64\|x\|^{2}}{2}=32\|x\|^{2} \geq \frac{1}{8}>c_{2} .
\end{aligned}
$$

Thus, all the assumptions of Corollary 10 hold. Then, we know that the considered functional difference system has at least three nonnegative 2-periodic solutions.

\section{Conflict of Interests}

The authors declare that there is no conflict of interests regarding the publication of this paper.

\section{Acknowledgments}

Hui-Sheng Ding acknowledges support from the NSF of China (11101192), the Program for Cultivating Young Scientist of Jiangxi Province (20133BCB23009), and the NSF of Jiangxi Province.

\section{References}

[1] B. Liu, "The existence and uniqueness of positive periodic solutions of Nicholson-type delay systems," Nonlinear Analysis: Real World Applications, vol. 12, no. 6, pp. 3145-3151, 2011.

[2] B. Liu and S. Gong, "Periodic solution for impulsive cellar neural networks with time-varying delays in the leakage terms," Abstract and Applied Analysis, vol. 2013, Article ID 701087, 10 pages, 2013.

[3] J. O. Alzabut and C. Tunç, "Existence of periodic solutions for Rayleigh equations with state-dependent delay," Electronic Journal of Differential Equations, vol. 77, pp. 1-8, 2012. 
[4] J. O. Alzabut, "Existence of periodic solutions for a type of linear difference equations with distributed delay," Advances in Difference Equations, p. 2012, article 53, 2012.

[5] H.-S. Ding and J. G. Dix, "Multiple Periodic Solutions for Discrete Nicholson's Blowflies Type System," Abstract and Applied Analysis, vol. 2014, Article ID 659152, 6 pages, 2014.

[6] J. G. Dix, S. Padhi, and S. Pati, "Multiple positive periodic solutions for a nonlinear first order functional difference equation," Journal of Difference Equations and Applications, vol. 16, no. 9, pp. 1037-1046, 2010.

[7] W. Long, X. J. Zheng, and L. Li, "Existence of periodic solutions for a class of functional integral equations," Electronic Journal of Qualitative Theory of Differential Equations, vol. 57, pp. 1-11, 2012.

[8] Y. N. Raffoul, "Positive periodic solutions of nonlinear functional difference equations," Electronic Journal of Differential Equations, vol. 55, pp. 1-8, 2002.

[9] Y. N. Raffoul and C. C. Tisdell, "Positive periodic solutions of functional discrete systems and population models," Advances in Difference Equations, no. 3, pp. 369-380, 2005.

[10] E. Braverman and S. H. Saker, "Permanence, oscillation and attractivity of the discrete hematopoiesis model with variable coefficients," Nonlinear Analysis: Theory, Methods \& Applications, vol. 67, no. 10, pp. 2955-2965, 2007.

[11] E. Braverman and S. H. Saker, "Periodic solutions and global attractivity of a discrete delay host macroparasite model," Journal of Difference Equations and Applications, vol. 16, no. 7, pp. 789-806, 2010.

[12] E. Braverman and S. H. Saker, "On the Cushing-Henson conjecture, delay difference equations and attenuant cycles," Journal of Difference Equations and Applications, vol. 14, no. 3, pp. 275-286, 2008.

[13] E. Braverman and S. H. Saker, "On a difference equation with exponentially decreasing nonlinearity," Discrete Dynamics in Nature and Society, vol. 2011, Article ID 147926, 17 pages, 2011.

[14] E. M. Elabbasy and S. H. Saker, "Periodic solutions and oscillation of discrete non-linear delay population dynamics model with external force," IMA Journal of Applied Mathematics, vol. 70, no. 6, pp. 753-767, 2005.

[15] S. H. Saker, "Qualitative analysis of discrete nonlinear delay survival red blood cells model," Nonlinear Analysis: Real World Applications, vol. 9, no. 2, pp. 471-489, 2008.

[16] S. H. Saker, "Periodic solutions, oscillation and attractivity of discrete nonlinear delay population model," Mathematical and Computer Modelling, vol. 47, no. 3-4, pp. 278-297, 2008.

[17] Z. C. Hao, T. J. Xiao, and J. Liang, "Multiple positive periodic solutions for delay differential system," Discrete Dynamics in Nature and Society, vol. 2009, Article ID 239209, 18 pages, 2009.

[18] R. I. Avery and J. Henderson, "Two positive fixed points of nonlinear operators on ordered Banach spaces," Communications on Applied Nonlinear Analysis, vol. 8, no. 1, pp. 27-36, 2001.

[19] R. W. Leggett and L. R. Williams, "Multiple positive fixed points of nonlinear operators on ordered Banach spaces," Indiana University Mathematics Journal, vol. 28, no. 4, pp. 673-688, 1979. 


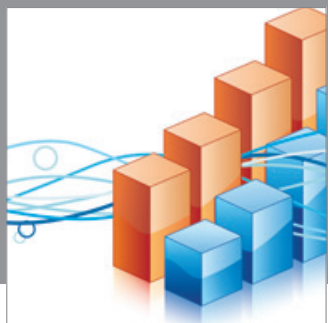

Advances in

Operations Research

mansans

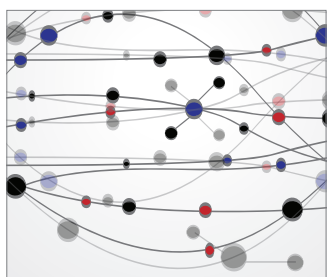

The Scientific World Journal
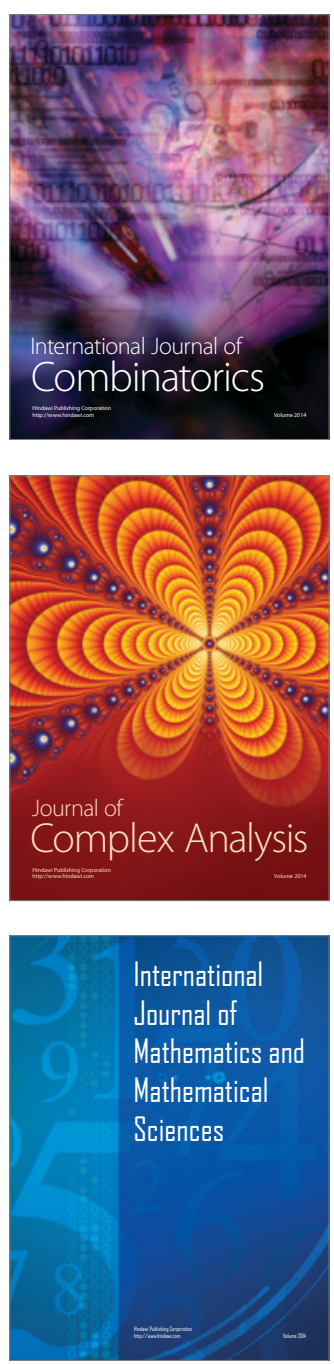
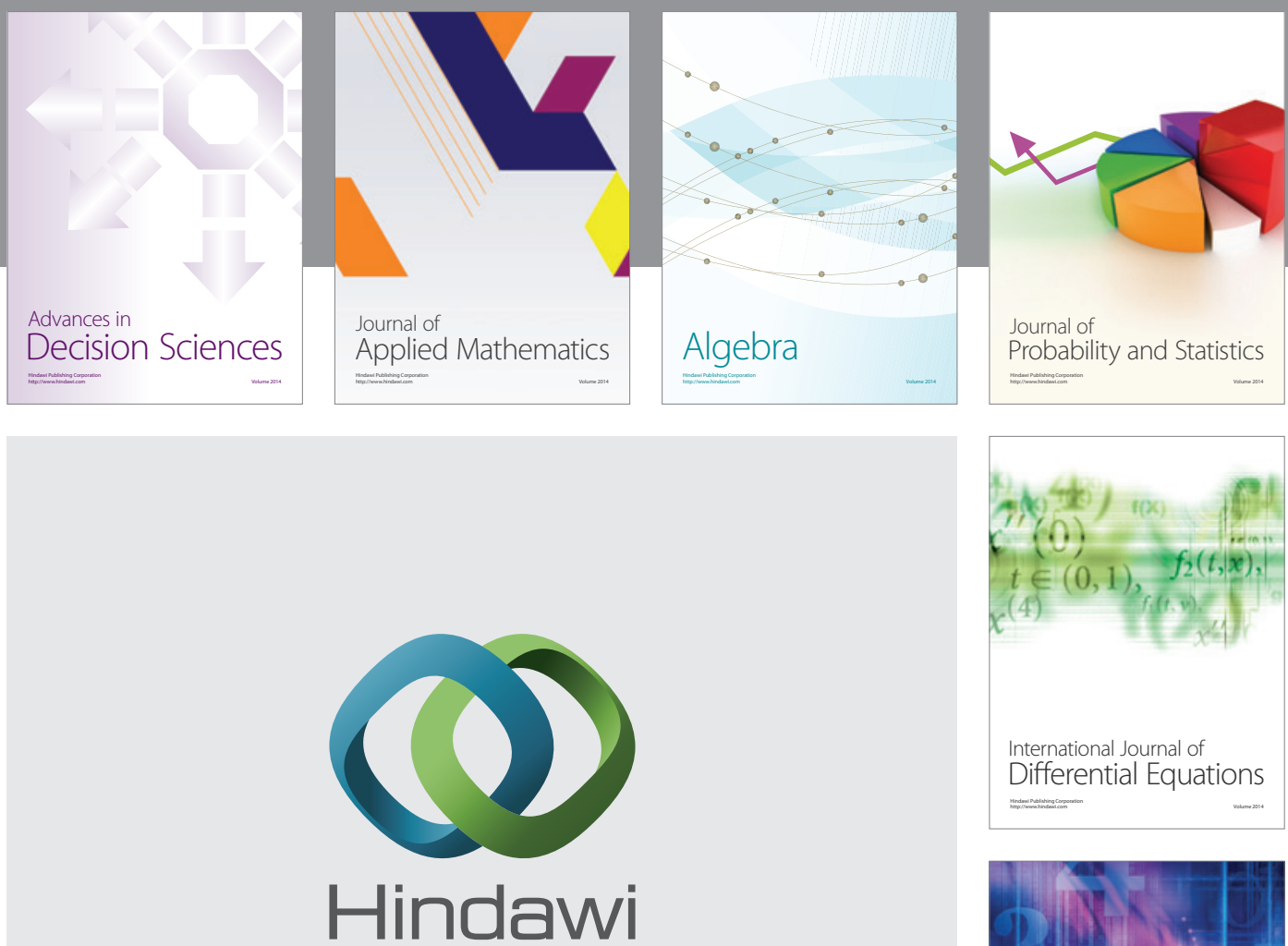

Submit your manuscripts at http://www.hindawi.com
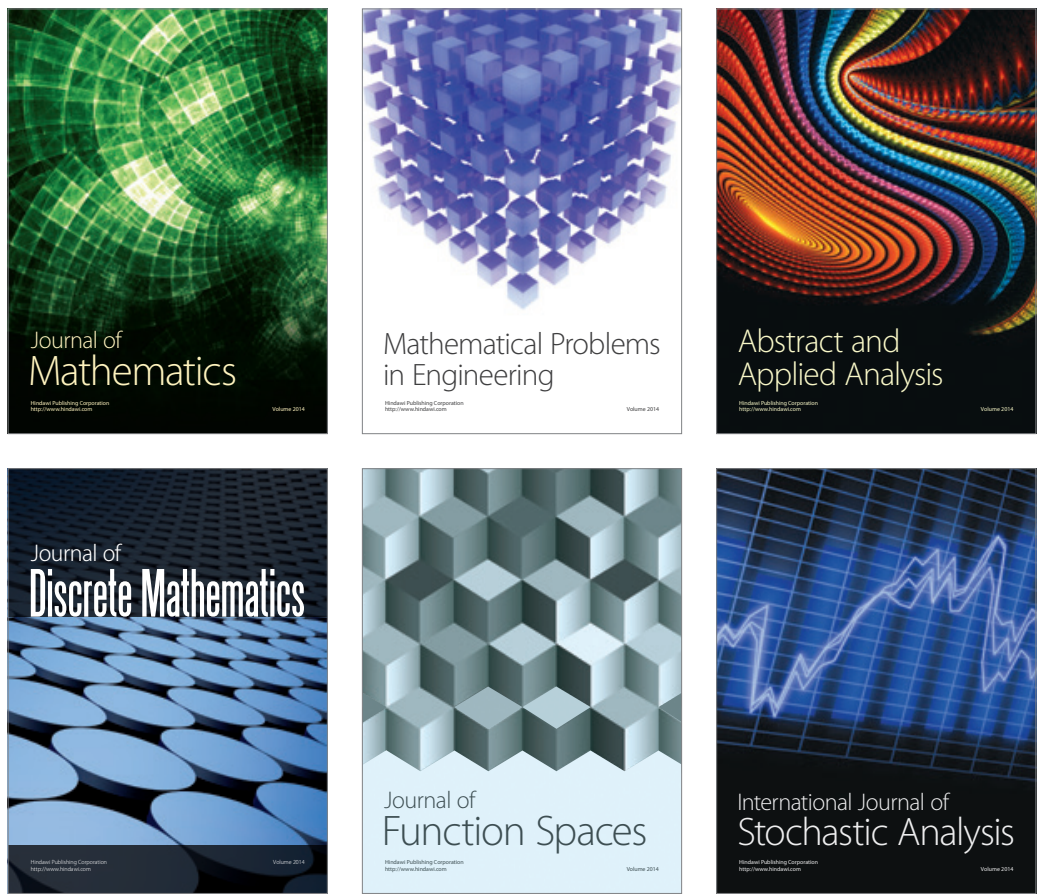

Journal of

Function Spaces

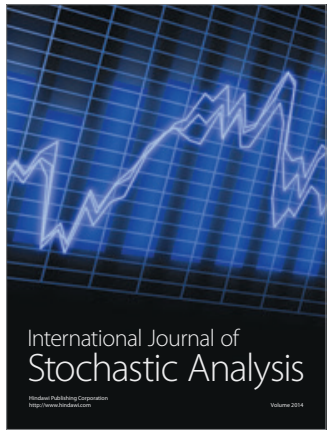

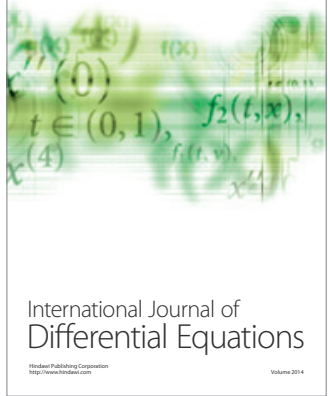
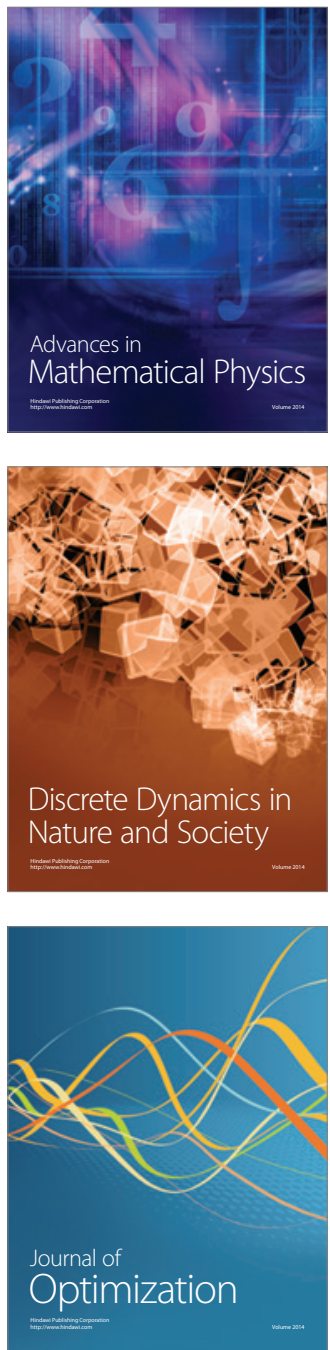